\title{
Improved Data Analysis with IVAS 4 and AP Suite
}

\author{
D.A. Reinhard ${ }^{1}$, T.R. Payne ${ }^{1}$, E.M. Strennen ${ }^{1}$, E. Oltman ${ }^{1}$, B.P. Geiser ${ }^{1}$, G.S. Sobering ${ }^{1}$, J. Mandt ${ }^{1}$ \\ 1. CAMECA Instruments Inc., 5470 Nobel Drive, Madison, WI 53711 USA. \\ * Corresponding author: David.reinhard@ametek.com
}

$\mathrm{CAMECA}^{\circledR}$ is in the process of re-structuring its $\mathrm{ISdb}^{\mathrm{TM}}$, IVAS ${ }^{\mathrm{TM}}$, and DAVis ${ }^{\mathrm{TM}}$ programs for atom probe tomography (APT). The currently separate programs are being brought together as modules within a new AP Suite $^{\mathrm{TM}}$. This new suite not only enables data transfer between the modules, but several new features that provide clear improvements to existing APT analyses.

AP Suite introduces three new mass spectrum analysis features to improve analysis accuracy: spatial ranging, and automated peak ranging and identification. Currently, IVAS utilizes a single set of global peak definitions which greatly limits interpretation of mass spectrum. Incorrect peak definitions for multiphase materials are difficult to avoid when a single peak range requires different definitions for each phase. Spatial ranging allows range definitions to be changed for multiple, user-defined regions of interest within a data set. This results in more accurate compositional analyses, as shown in Fig. 1. In addition, two important sources of analysis variability result from analyst-dependent peak identification and peak ranging processes [1]. In AP Suite, auto peak-identification, based on Bayesian statistical analysis of the spectrum [2], and auto range-limit generation, are designed to further reduce analyst-dependent variation.

APT experiments are controlled by automated voltage adjustment to maintain a constant ion detection rate. This is a simple and effective method, but additional controls, such as adjusting the laser pulse energy to maintain a constant charge-state-ratio, have been created which rely on analysis of the mass spectrum. AP Suite contains new functionality within the DAVis program to correct and actively calibrate the mass spectrum during an acquisition. This improves the capability of control algorithms, like the constant charge-state-ratio, and opens the door to more advanced control schemes based on detection of compositionally-distinct features [3].

New display features are also available with AP Suite. Specimens analyzed by APT are often imaged with correlative techniques [4]. Overlay of the 3D APT data with the 2D images from other techniques allow for direct comparisons and deeper insights into the nature of the materials under investigation (see Fig. 2).

IVAS $4^{\mathrm{TM}}$ introduces a new APT file format; an extensible file format which allows files to be created with only the desired information and allows new types of information to be defined and included in the future without breaking existing tools. The most common file format for reconstructed APT data is the POS format. This is a simple binary file consisting of the $\mathrm{X}, \mathrm{Y}$ and $\mathrm{Z}$ coordinates, and the mass-to-charge value of each ion [5]. At times, additional information is required to enable novel analyses [6]. The EPOS and ATO file formats contain additional records for each ion, but they require the generation of a larger data file than may be needed [5]. The APT format allows for inclusion of new ion information to enable new analyses in the future, without changing the format and breaking existing tools.

The IVAS 4 module in AP Suite allows external tools to be plugged into the analysis tree to execute a custom analysis algorithm on the data. These external tools can access any data in the APT file and will 
be able to write new data into the APT file [7]. This enables more experimentation with case-specific analyses and facilitates sharing of analysis tools across the atom probe community.

\section{References:}

[1] F Exertier et al., Chemical Geology 495 (2018), p. 27.

[2] A Mikhalychev and A. Ulyanenkov, Journal of Applied Crystallography 50 (2017), p. 776.

[3] TJ Prosa et al., Front Char. and Met. for Nanoelec. (NIST, Gaithersburg, Md, 2013), pp. 269-272.

[4] Y Chen et al., Microscopy and Microanalysis. 22 (2016), p. 682.

[5] DJ Larson et al., Local Electrode Atom Probe Tomography: A User's Guide. New York: Springer, (2013), pp. 249-256.

[6] DW Saxey, Ultramicroscopy 111 (2011), pp. 473-479.

[7] AC Day et al., Recent Developments in APT Analysis Automation and Support for User-Defined Custom Analysis Procedures in IVAS 4.0 (This Proceeding).
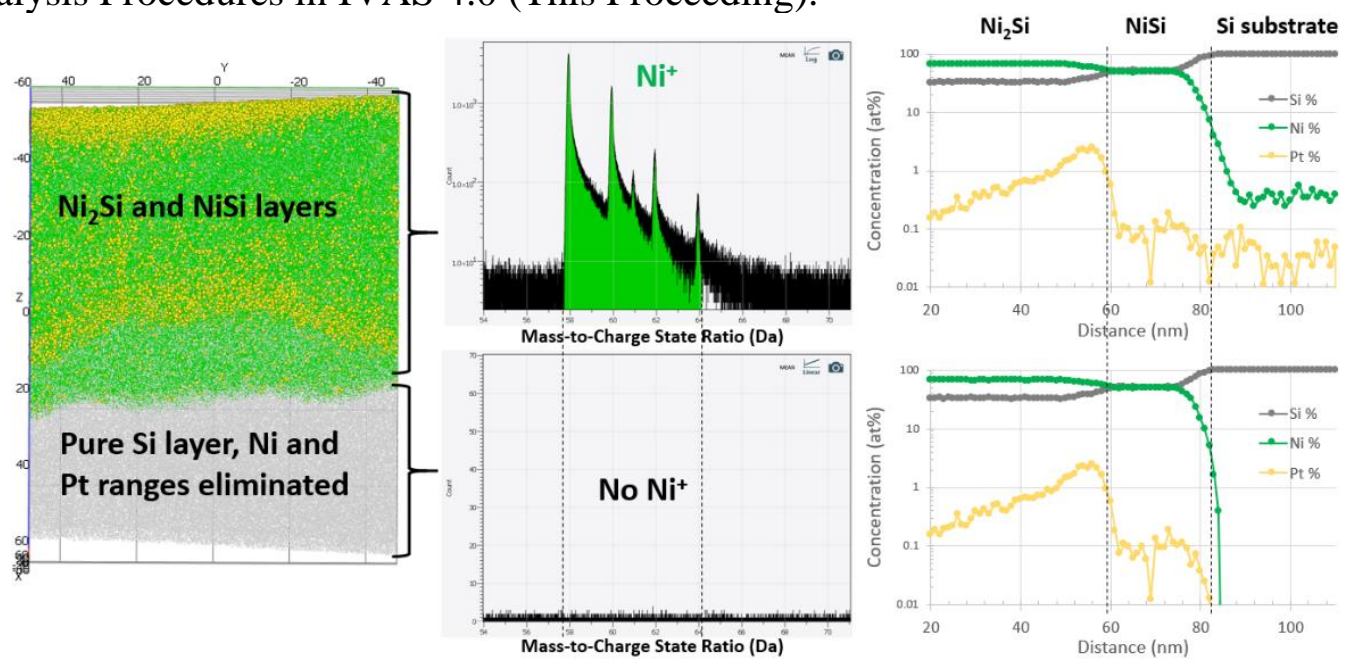

Figure 1. An example of spatial ranging benefits. (left) An atom map of a NiSi/Si interface. (middle) Mass spectra from each major phase showing the presence and absence of Ni. (right) 1D composition profiles showing that by minimizing peak overlaps, the true $\mathrm{Ni}$ and Pt composition profiles (top to bottom of atom map) are more accurately represented.

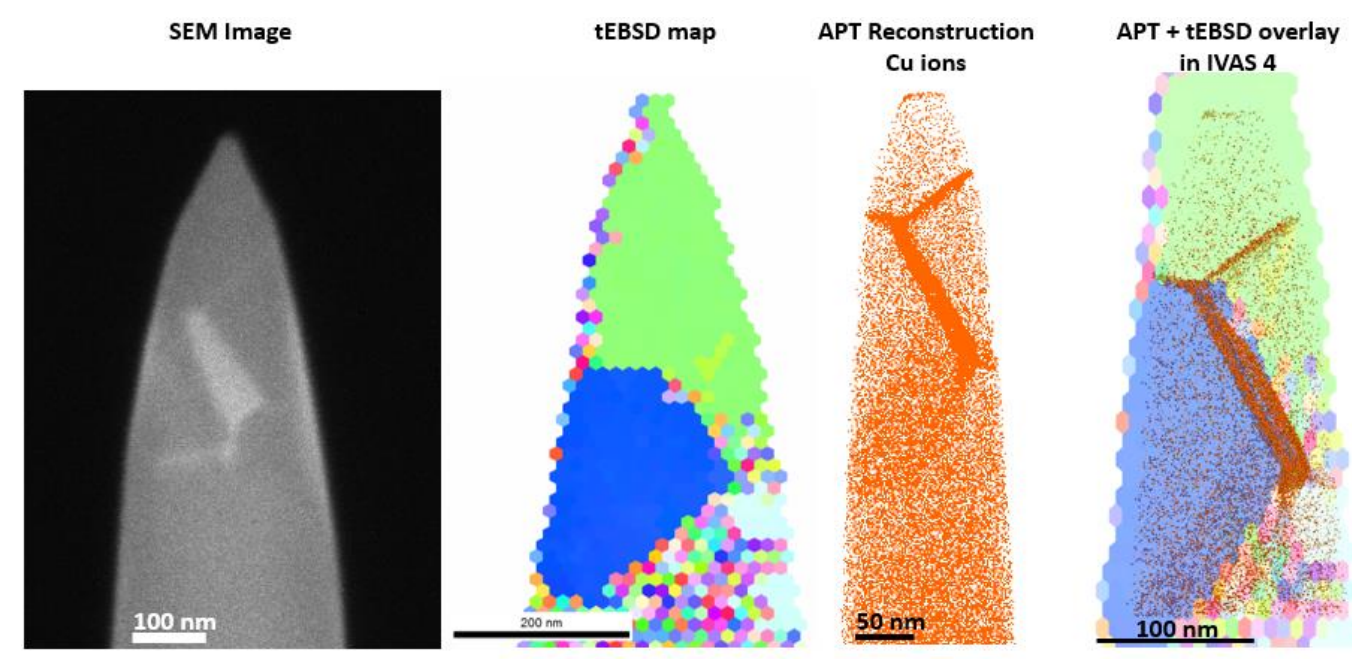

Figure 2. Example image overlay feature in IVAS 4. Here a set of correlative images (SEM, tEBSD, APT) of a single specimen are shown. Any combination of images can be combined for 3D display. 